\title{
RECURRENCE RELATIONS FOR PROLATE SPHEROIDAL WAVE FUNCTIONS
}

\section{IMANUEL MARX}

1. Introduction. Recent investigations of electromagnetic problems in regions bounded by spheroids have led to an increased interest in the spheroidal wave functions. These functions $S_{m n}(c, \eta), R_{m n}(c, \xi)$ result from the solution of the scalar wave equation by separation in spheroldal coordinates The properties of $S_{m n}$ and $R_{m n}$ are discussed in [1] and [6] The notation throughout this paper will follow that of [1].

Recurrence relations among the spheroidal wave functions cannot be established by the methods for functions of hypergeometric type, because the differential equation satisfied by these functions has an irregular singular point at infinity in addition to regular singular points for the values \pm 1 of the respective variable. However, a method suggested by E. T. Whittaker [7], but apparently not fully exploited by him, leads to recurrence relations for the radial functions $R_{m n}(c, \xi)$ as well as for the non-integral separation constants that appear in the differential equations of both the radial and the angular functions. Formulas are obtained in detail for the prolate spheroidal wave functions. A simple substitution $[4$, p. 151] formally gives the corresponding relations for the oblate spheroidal functions, and the validity of these relations can be established by a suitable modification of the proof.

Whittaker observes that in the case of the two-dimensional wave equation $\partial^{2} u / \partial x^{2}+\partial^{2} u / \partial y^{2}+u=0$ the derivative $\partial u / \partial x$ of a solution $u(x, y)$ is likewise a solution. After separation of the equation in polar coordinates, $\partial u / \partial x$ can be expanded in a series of separated solutions $J_{n}(r) \cos n \theta$. Write $\partial u / \partial x=\cos \theta \partial u / \partial r-r^{-1} \sin \theta \partial u / \partial \theta$, and equate to the series of Bessel functions. The resulting identity, when multiplied by $\cos p \theta$ and integrated from $-\pi$ to $\pi$, yields recurrence relations for the Bessel functions in terms of the coefficients in the expansion of $\partial u / \partial x$ Whittaker does not show how to obtain these coefficients. In the present three dimensional case, the analogous coefficients are represented as integrals of products of the spheroidal wave functions. Since these integrals involve the functions whose recurrence relations are being investigated, the existence of these relations and their nature, rather than a practically applicable form, are demonstrated. For practical applications, an evaluation of the coefficients by some independent method (such as is furnished for many known functions by the hypergeometric series) would seem to be necessary. It is hoped that further investigation will lead to such a method.

Procedures similar to those used in this paper may lead to recurrence relations in other cases of separable partial differential equations with constant coefficients. As long as the separated equations are of Sturm-Liouville type, and the solutions can be shown to have suitable orthogonality properties, an analogous method may be developed. 
2. Properties of Wave Functions. The solutions $\Psi(x, y, z)$ of the scalar wave equation

$$
\frac{\partial^{2} \Psi}{\partial x^{2}}+\frac{\partial^{2} \Psi}{\partial y^{2}}+\frac{\partial^{2} \Psi}{\partial z^{2}}+k^{2} \Psi=0
$$

are to be obtained by a separation of the form

$$
\Psi(x, y, z)=\Omega(\eta, \xi, \phi)=S(\eta) R(\xi) \Phi(\phi),
$$

where

$$
\begin{aligned}
& x=\frac{1}{2} d\left(1-\eta^{2}\right)^{\frac{1}{3}}\left(\xi^{2}-1\right)^{\frac{1}{2}} \cos \phi, \\
& y=\frac{1}{2} d\left(1-\eta^{2}\right)^{\frac{1}{2}}\left(\xi^{2}-1\right)^{\frac{1}{2}} \sin \phi, \\
& z=\frac{1}{2} d \eta \xi .
\end{aligned}
$$

The surfaces $\eta=$ const., $-1 \leqq \eta \leqq 1$, are confocal hyperboloids of revolution of two sheets, the surfaces $\xi=$ const., $1 \leqq \xi<\infty$, are confocal prolate spheroids, and $d$ is the focal distance common to both sets of surfaces. The surfaces $\phi=$ const., $0 \leqq \phi<2 \pi$, are half-planes containing the $z$-axis and terminated by it.

In terms of the spheroidal coordinates, equation (1) becomes

$$
\begin{aligned}
\frac{\partial}{\partial \eta}\left(1-\eta^{2}\right) \frac{\partial \psi}{\partial \eta} & +\frac{\partial}{\partial \xi}\left(\xi^{2}-1\right) \frac{\partial \psi}{\partial \xi}+\frac{\left(\xi^{2}-\eta^{2}\right)}{\left(\xi^{2}-1\right)\left(1-\eta^{2}\right)} \frac{\partial^{2} \psi}{\partial \varphi^{2}} \\
& +c^{2}\left(\xi^{2}-\eta^{2}\right) \psi=0,
\end{aligned}
$$

where $c=\frac{1}{2} k d$. The separation of $\Phi(\phi)$ leads to the equation $\Phi^{\prime \prime}+m^{2} \Phi=0$. The separation constant $m$ must be integral or zero to satisfy the condition of periodicity. Further separation gives a separation constant $\lambda_{m n}$ and the differential equations

$$
\begin{aligned}
& \frac{d}{d \eta}\left(1-\eta^{2}\right) \frac{d S_{m n}}{d \eta}-\left(\frac{m^{2}}{1-\eta^{2}}-\lambda_{m n}+c^{2} \eta^{2}\right) S_{m n}=0 \\
& \frac{d}{d \xi}\left(\xi^{2}-1\right) \frac{d R_{m n}}{d \xi}-\left(\frac{m^{2}}{\xi^{2}-1}+\lambda_{m n}-c^{2} \xi^{2}\right) R_{m n}=0 .
\end{aligned}
$$

The separation constant is chosen so as to ensure that the solution of equation (2) which is free of logarithmic terms is regular in the entire range $-1 \leqq \eta \leqq 1$. These solutions form, for given $m$, a countable sequence of "angular wave functions of the first kind" $S_{m n}^{(1)}(c, \eta), n=0,1, \ldots$ The solutions of (3) for the same values of $\lambda_{m n}$ and which are also free of logarithmic terms are chosen as the "radial wave functions of the first kind" $R_{m n}^{(1)}(c, \xi)$. Since only functions of the first kind will be treated here, and since the parameter $c$ will remain fixed in the discussion, the superscript (1) and the argument $c$ will be omitted. The properties of these functions are easily deduced from explicit series expansions given in [1]. Page references to this report are given below

The angular wave functions have the following behavior at the end points of their interval of definition: as $n \rightarrow 1$, 
(4)

$$
\begin{gathered}
S_{m n}(\eta)=0(1-\eta), d S_{m n}(\eta) / d \eta=0(1), m \neq 0,1 \\
S_{o n}(\eta)=0(1), d S_{o n}(\eta) / d \eta=0(1) \\
S_{l n}(\eta)=(1-\eta)^{\frac{1}{2}}\left[a_{n}+0(1-\eta)\right], \quad d S_{l n}(\eta) / d \eta \sim b_{n}(1-\eta)^{-\frac{1}{2}}
\end{gathered}
$$

[p. 32]. The behavior as $\eta \rightarrow-1$ is analogous, with $1-\eta$ replaced by $1+\eta$.

The radial functions behave as follows at the finite end of their interval: as $\xi \rightarrow 1$,

$$
\begin{gathered}
R_{m n}(\xi)=0(\xi-1), \quad d R_{m n}(\xi) / d \xi=0(1), \quad m \neq 0,1 \\
R_{o n}(\xi)=0(1), \quad d R_{o n}(\xi) / d \xi=0(1) ;
\end{gathered}
$$

$R_{1 n}(\xi)=(\xi-1)^{\frac{1}{1}}\left[a_{n}^{\prime}+0(\xi-1)\right], \quad d R_{1 n}(\xi) / d \xi \sim(\xi-1)^{-\frac{1}{2}}\left[b_{n}^{\prime}+0(\xi-1)\right]$ [pp. 34-35] As $\xi \rightarrow \infty$, the asymptotic behavior is described by:

$$
R_{m n}(\xi) \sim(c \xi)^{-1} \cos \left[c \xi-\frac{1}{2}(n+1) \pi\right]
$$

$$
d R_{m n}(\xi) / d \xi \sim-c(c \xi)^{-1} \sin \left[c \xi-\frac{1}{2}(n+1) \pi\right]
$$

[p. 24.]

The existence of the integrals

$$
\int_{1}^{\infty} R_{m n}(\xi) R_{m p}(\xi) d \xi
$$

is easily established from (5) and (6). For $n \neq p$, the standard Sturm-Liouville method leads to the partial orthogonality relations

$$
\int_{1}^{\infty} R_{m n}(\xi) R_{m p}(\xi) d \xi=\left\{\begin{array}{l}
0, \quad n-p \text { even, } \\
(-1)^{(n-p-1) / 2} / c\left(\lambda_{m n}-\lambda_{m p}\right), n-p \text { odd }
\end{array}\right\}
$$

[cf. 3, p. 237-238], the behavıor at infinity detailed in (6) gives the stated values for the integrated term. The case $n=p$ gives the normalization integral

$$
\int_{1}^{\infty} R_{m n}^{2}(\xi) d \xi=M_{m n}
$$

Similarly, one finds that

$$
\int_{-1}^{1} S_{m n}(\eta) S_{m p}(\eta) d \eta=\delta_{n}^{p} N_{m n}
$$

where $\delta_{n}^{p}$ is the Kronecker $\delta$ symbol.

3. Expansion of Solutions. The methods described in $[2, \mathrm{Ch} . \mathrm{IV}]$ or $[5, \mathrm{Ch}$. VI, \$4], and in [3, Ch. XI], show that an arbitrary regular solution of the scalar wave equation may be expressed in a termwise integrable double series of the functions

$$
\psi_{m n}(\eta, \xi, \phi)=S_{m n}(\eta) R_{m n}(\xi) \cos m \phi
$$


and

$$
\omega_{m n}(\eta, \xi, \phi)=S_{m n}(\eta) R_{m n}(\xi) \sin m \phi .
$$

Write $\psi_{m n}(\eta, \xi, \phi)=\Psi_{m n}(x, y, z)$. The functions $\partial \Psi_{m n} / \partial x, \partial \Psi_{m n} / \partial y, \partial \Psi_{m n} / \partial z$ are continuous solutions of the scalar wave equation (1). The derivatives $\partial \Psi_{m n} / \partial x$ and $\partial \Psi_{m n} / \partial z$ are even functions of $\phi$ and may be expanded in a series of functions $\Psi_{\mu \nu}$, while $\partial \Psi_{m n} / \partial y$ is an odd function of $\phi$ and may be expanded in a series of functions $\omega_{\mu \nu}$. The first set of formulas will be obtained in detail from the expansion

$$
\partial \Psi_{m n} / \partial z=\sum_{\mu=0}^{\infty} \sum_{\nu=o}^{\infty} g_{\mu \nu}^{m n} \psi_{\mu \nu}(\eta, \xi, \phi) .
$$

For the other two sets of formulas only an indication of certain modifications of the method and the final results will be given.

Expansion of the left member of (10) gives:

$$
\begin{aligned}
{\left[\xi\left(1-\eta^{2}\right) S_{m n}^{\prime} R_{m n} \cos m \phi+\eta\left(\xi^{2}-1\right) S_{m n} R_{m n}^{\prime} \cos m \phi\right] / d\left(\xi^{2}-\eta^{2}\right) } \\
=\sum_{\mu=0}^{\infty} \sum_{\nu=0}^{\infty} g_{\mu \nu}^{m n} S_{\mu \nu}(\eta) R_{\mu \nu}(\xi) \cos \mu \phi .
\end{aligned}
$$

Multiply both members of (11) by $\cos p \phi$ and integrate from 0 to $2 \pi$. On the left there is a non-zero result only for $p=m$, on the right only for $p=\mu$ :

$$
0=\sum_{\nu=o}^{\infty} g_{p \nu}^{m n} S_{p \nu}(\eta) R_{p \nu}(\xi), \quad p \neq m
$$

and

$$
\begin{aligned}
{\left[\xi\left(1-\eta^{2}\right) S_{m n}^{\prime} R_{m n}+\eta\left(\xi^{2}-1\right) S_{m n} R_{m n}^{\prime}\right] / d\left(\xi^{2}\right.} & \left.-\eta^{2}\right) \\
& =\sum_{\nu=0}^{\infty} g_{m \nu}^{m n} S_{m \nu} R_{m \nu}
\end{aligned}
$$

Next multiply by $S_{p \kappa}(\eta)$ or $S_{m \kappa}(\eta)$, respectively, integrate from -1 to 1 with respect to $\eta$, and apply (9). From (12) one obtains

$$
g_{p \nu}^{m n}=0, \quad p \neq m,
$$

i.e. in the expansion of $\partial \Psi_{m n} / \partial z$ only the functions $\psi_{m \nu}$ need have been used. In (13) there is a non-zero result on the right only for $\kappa=\nu$. The left member is the sum of the integrals

$$
\begin{aligned}
I_{\nu}^{m n}(\xi) & =\frac{2}{d} \int_{-1}^{1} R_{m n}(\xi) \xi \frac{1-\eta^{2}}{\xi^{2}-\eta^{2}} S_{m n}^{\prime}(\eta) S_{m \nu}(\eta) d \eta \\
J_{\nu}^{m n}(\xi) & =\frac{2}{d} \int_{-1}^{1} R_{m n}^{\prime}(\xi) \eta \frac{\xi^{2}-1}{\xi^{2}-\eta^{2}} S_{m n}(\eta) S_{m \nu}(\eta) d \eta .
\end{aligned}
$$

The existence of these integrals must be demonstrated. For $m \neq 1$, the only difficulty which might arise comes from the functions $\left(1-\eta^{2}\right) /\left(\xi^{2}-\eta^{2}\right)$ and $\left(\xi^{2}-1\right) /\left(\xi^{2}-\eta^{2}\right)$ near $\eta=1, \xi=1$, or $\eta=-1, \xi=1$. However, since the functions are bounded near these points, the integrals exist. For $m=1$, by virtue of (4), (5) the integrand of $I$ near $\eta=1, \xi=1$ may be written as:

$$
b_{n} \xi(\xi-1)^{\frac{1}{2}}\left[a_{n}^{\prime}+0(\xi-1)\right] \frac{1-\eta}{\xi-\eta} \frac{1+\eta}{\xi+\eta}\left[a_{\nu}+0(1-\eta)\right]
$$


while the integrand of $J$ has the form:

$$
(\xi-1)^{\frac{1}{2}}\left[b_{n}^{\prime}+0(\xi-1)\right] \eta \frac{\xi+1}{\xi+\eta} \frac{1-\eta}{\xi-\eta}\left[a_{n} a_{\nu}+0(1-\eta)\right]
$$

Similar estimates hold near $\eta=-1, \xi=1$. The integrands are again seen to be bounded, and the integrals converge.

The series representations in [1, p. 32] show that the integrands in the integrals $I, J$, are odd functions of $\eta$ for $n-m$ and $\nu-m$ both even or both odd, and the coefficients $g_{m \nu}^{m n}$ are accordingly zero when $\nu-n$ is an even integer or zero.

4. Recurrence Relations for Radial Functions. The formulas obtained can now be written as

$$
\begin{aligned}
(2 / d) \int_{-1}^{1}\left(\xi^{2}-\eta^{2}\right)^{-1}\left[\xi\left(1-\eta^{2}\right) S_{m n}^{\prime}(\eta) S_{m \nu}(\eta) R_{m n}(\xi)\right. & \\
& \left.+\eta\left(\xi^{2}-1\right) S_{m n}(\eta) S_{m \nu}(\eta) R_{m n}^{\prime}(\xi)\right] d \eta \\
& =g_{m \nu}^{m n} N_{m \nu} R_{m \nu}(\xi)
\end{aligned}
$$

for $n=0,1, \cdots$, and for $\nu-n$ odd. The relations in (15) display $R_{m \nu}$ as a linear combination of $R_{m n}$ and $R_{m n}^{\prime}$, with coefficients that are continuous functions of $\xi$ in any finite subinterval of the range $1 \leqq \xi<\infty$.

For evaluation of the constant $g_{m \nu}^{m n}$, an integration over $\xi$ is required. For large $\xi$, the significant factors in $I_{v}^{m n}$ are

$$
\left(\xi^{2}-\eta^{2}\right)^{-1} \xi R_{m n}(\xi)=1 / \xi\left(1-\eta^{2} / \xi^{2}\right)^{-1} R_{m n}(\xi),
$$

while those in $J_{v}^{m n}$ are

$$
\left(\xi^{2}-1\right)\left(\xi^{2}-\eta^{2}\right)^{-1} R_{m n}^{\prime}(\xi)=\left(1-\eta^{2} / \xi^{2}\right)^{-1}\left(1-1 / \xi^{2}\right) R_{m n}^{\prime}(\xi) .
$$

It follows from (6) that the integrals converge at infinity. Near $\xi=1$, the estimates established previous to the integration with respect to $\eta$ are adequate to prove the existence of the integrals. The integration yields

$$
\begin{gathered}
g_{m \nu}^{m n}=2 / d N_{m \nu} M_{m \nu} \int_{1}^{\infty} \int_{-1}^{1}\left(\xi^{2}-\eta^{2}\right)^{-1}\left[\xi\left(1-\eta^{2}\right) R_{m n} R_{m \nu} S_{m n}^{\prime} S_{m \nu}\right. \\
\left.+\eta\left(\xi^{2}-1\right) R_{m n}^{\prime} R_{m \nu} S_{m n} S_{m \nu}\right] d \eta d \xi
\end{gathered}
$$

Alternatively, after dividing both sides of (15) by $N_{m \nu} R_{m \nu}(\xi)$ one may note that the right hand member is constant. Therefore the left hand member is independent of $\xi$, and substitution of a convenient value $\xi_{0}$ gives $g_{m \nu}^{m n}$ in terms of the integral over $\eta$.

5. Recurrence Relations for Index $m$. Relations connecting the radial functions $R_{m n}(\xi), R_{m n}^{\prime}(\xi)$, and $R_{m \pm 1, \nu}(\xi)$ are obtained by an application of the method to the expansion of $\partial \Psi_{m n} / \partial x$ in functions $\psi_{\mu \nu}(\eta, \xi, \phi)$. The expansion of $\partial \Psi_{m n} / \partial y$ in functions $\omega_{\mu \nu}(\eta, \xi, \phi)$ would lead to the same relations Similarly to (11), the expansions give 


$$
\begin{gathered}
\partial \Psi_{m n} / \partial x=\sum_{\mu=0}^{\infty} \sum_{\nu=0}^{\infty} h_{\mu \nu}^{m n} \psi_{\mu \nu} \\
=(2 T / d D)\left[-\eta S_{m n}^{\prime} R_{m n}+\xi S_{m n} R_{m n}^{\prime}\right] \cos \phi \cos m \phi \\
+(2 m / d T) S_{m n} R_{m n} \sin \phi \sin m \phi
\end{gathered}
$$

where

$$
D=\xi^{2}-\eta^{2}, T=\left(1-\eta^{2}\right)^{\frac{1}{2}}\left(\xi^{2}-1\right)^{\frac{1}{2}} .
$$

Trigonometric transformation changes the right hand members to linear combinations of $\cos (m \pm 1) \phi$, and multiplication by $\cos p \phi$ and integration with respect to $\phi$ from 0 to $2 \pi$ eliminates one summation:

$$
\begin{gathered}
\sum_{\nu=0}^{\infty} h_{p \nu}^{m n} S_{p \nu} R_{p \nu}=0, \quad m>0, \quad p \neq m \pm 1 ; \\
\sum_{\nu=0}^{\infty} h_{p \nu}^{o n} S_{p \nu} R_{p \nu}=0, \quad p \neq 1,
\end{gathered}
$$

and

$$
\begin{aligned}
\sum_{\nu=0}^{\infty} h_{m \pm 1, \nu}^{m n} S_{m \pm 1, \nu} R_{m \pm 1, \nu} & =\mp \frac{T}{d D}\left(\eta S_{m n}^{\prime} R_{m n}-\xi S_{m n} R_{m n}^{\prime}\right) \\
& -\frac{m}{d T} S_{m n} R_{m n}, \quad m>0 \\
\sum_{r=0}^{\infty} h_{1 \nu}^{o n} S_{1 v} R_{1 \nu} & =-\frac{2 T}{d D}\left(\eta S_{o n}^{\prime} R_{o n}-\xi S_{o n} R_{o n}^{\prime}\right)
\end{aligned}
$$

The procedure of section 3 gives the following recurrence relations for the radial functions:

$$
\begin{gathered}
h_{m \pm 1, \nu}^{m n} N_{m \pm 1, \nu} R_{m \pm 1, \nu}=\mp(1 / d) \int_{-1}^{1}(T / D)\left(\eta S_{m n}^{\prime} R_{m n}-\xi S_{m n} R_{m n}^{\prime}\right) S_{m \pm 1, \nu} d \eta \\
-(m / d) \int_{-1}^{1}(1 / T) S_{m n} R_{m n} d \eta, \quad m>0 \\
h_{1 \nu}^{o n} N_{t \nu} R_{1 \nu}=-(2 / d) \int_{-1}^{1}(T / D)\left(\eta S_{o n}^{\prime} R_{o n}-\xi S_{o n} R_{o n}^{\prime}\right) S_{m \pm 1, \nu} d \eta
\end{gathered}
$$

These relations are valid for $n=0,1, \cdots$, and for $n-\nu$ an odd integer. The coefficients corresponding to $\nu-n$ zero or even, as well as all coefficients not appearing in formulas (21), are zero. The evaluation of the non-zero coefficients $h_{\mu \nu}^{m n}$ may be carried out as suggested in section 4 .

6. Recurrence Relations for Separation Constants. Because of the incomplete orthogonality (7) of the radial functions, the procedure of the foregoing sections does not appear to yield recurrence relations for the angular functions $S_{m n}(\eta)$ However, a related procedure can be used to derive a recurrence relation for the (non-integral) separation constants $\lambda_{m n}$. Estimates similar to those in the discussions of the integrals (14) and (16) show that multiplication of both members 
of (13) by $R_{m \nu}(\xi)$ and integration with respect to $\xi$ from 1 to $\infty$ is a valid operation. The result is the formula

$$
\begin{aligned}
(2 / d) \int_{1}^{\infty}(1 / D) & {\left[\xi\left(1-\eta^{2}\right) S_{m n}^{\prime} R_{m n} R_{m \nu}+\eta\left(\xi^{2}-1\right) S_{m n} R_{m n}^{\prime} R_{m \nu}\right] d \xi } \\
= & g_{m \nu}^{m n} M_{m \nu} S_{m \nu}+\sum_{p=0,1}^{\prime \infty}(-1)^{(\nu-p-1) / 2} g_{m p}^{m n} S_{m p} / c\left(\lambda_{m p}-\lambda_{m v}\right),
\end{aligned}
$$

where $D=\xi^{2}-\eta^{2}$ as before, and where the summation symbol denotes a sum over even $p$ if $\nu$ is odd, a sum over odd $p$ if $\nu$ is even. Next both members are multiplied by $S_{m p}(\eta)$ and integrated with respect to $\eta$ from -1 to 1 to produce the relation

$$
d_{p \nu}^{m n}=(-1)^{(\nu-p-1) / 2} g_{m p}^{m n} N_{m p} / c\left(\lambda_{m p}-\lambda_{m v}\right),
$$

where $d_{p \nu}^{m n}$ stands for the integral of the left member of (22) with respect to $\eta$ The ratio of $d_{p \nu}^{m n}$ and $g_{m p}^{m n}$ is evidently independent of $n$. Writing

$$
c_{p \nu}^{m}=(-1)^{(\nu-p-1) / 2} g_{m p}^{m n} N_{m p} / c d_{p \nu}^{m n}
$$

one has finally

$$
\lambda_{m p}-\lambda_{m \nu}=c_{p \nu}^{m}
$$

for any untegers $p, \nu$ whose difference is an odd integer. More generally,

$$
\lambda_{m n}=\lambda_{m o}+\sum_{p=1}^{n} c_{p, p-1}^{m} .
$$

A similar procedure applied to the formulas of section 5 leads to no essentially different results.

Some further detals about the coefficients in the recurrence relations will appear in UMM 126, a forthcoming publication of the Willow Run Research Center.

\section{REFERENCES}

1. C Flammer, "Spheroidal Wave Functions," Technical Report, Stanford Research Institute, Stanford, 1951

2 B. Friedman, "Techniques in Solving Partial Differential Equations," Institute for Mathematıcs and Mechanics, New York Unıversity, 1950

3. E L INCE, "Ordinary Differential Equations," Dover, New York, 1944

4 E Madenung, "Die Mathematischen Hilfsmittel des Physikers," Dover, New York, 1943

5 P M Morse and H Feshbach, "Methods of Theoretical Physics," Technology Press, M I T, 1946.

6 J A Stratton, P. M Morse, L J Chu, and R A Hutner, "Elliptic Cylinder and Spheroidal Wave Functions," Wiley, New York, 1950

7. E T Whitraker, "Recurrence Formulae for Mathieu Functions," Jour L M S 4, Vol 88 (1929)

Willow Run Research Center of the University of Michigan

(Received January 12, 1953, Revised June 15, 1953) 